\title{
Neuropsicología del trastorno de Tourette: cognición, neuroimagen y creatividad
}

\author{
Raúl Espert, Marien Gadea, Marta Aliño, Javier Oltra-Cucarella
}

Introducción. El trastorno de Tourette es el resultado de una disfunción cerebral frontoestriatal que afecta a personas de todas las edades, con un inicio en la primera infancia y continuación en la adolescencia y la adultez.

Desarrollo. Este artículo revisa los principales aspectos cognitivos, de neuroimagen funcional y estudios relacionados con la creatividad en un trastorno caracterizado por un exceso de dopamina en el cerebro.

Conclusiones. Dada la especial configuración cerebral de estos pacientes, deberían esperarse alteraciones neuropsicológicas, especialmente en las funciones ejecutivas. Sin embargo, los hallazgos son poco concluyentes y están condicionados por factores como la comorbilidad con el trastorno por déficit de atención/hiperactividad y el trastorno obsesivo compulsivo, la edad o variables metodológicas. Por otro lado, los estudios de neuroimagen realizados a lo largo de la última década han podido explicar la sintomatología clínica de pacientes con trastorno de Tourette, con especial relevancia del área motora suplementaria y el giro cingulado anterior. Finalmente, a pesar de no existir una relación lineal entre el exceso de dopamina y la creatividad, la literatura científica destaca una asociación entre el trastorno de Tourette y la creatividad musical, lo que podría traducirse en programas de intervención basados en la música.

Palabras clave. Cognición. Creatividad. Dopamina. Neuroimagen funcional. Neuropsicología. Trastorno de Tourette.

\section{Aspectos cognitivos}

El trastorno de Tourette (TT) es un trastorno del neurodesarrollo que se diagnostica en la infancia o adolescencia temprana [1]. Los principales síntomas de TT incluyen múltiples tics motores y uno o más tics vocales, que duran más de un año. Los tics pueden ser de naturaleza simple o compleja y varían en número, frecuencia y gravedad en el tiempo. El fenotipo 'trastorno de Tourette sin comorbilidades' (TTSC), sin comorbilidad con otras condiciones clínicas, apenas supone el $10 \%$ de todos los TT [2]. Los trastornos comórbidos incluyen ansiedad, depresión y trastornos del aprendizaje y del sueño, aunque los más comunes son el trastorno por déficit de atención/hiperactividad (TDAH) y el trastorno obsesivo compulsivo (TOC) [3]. Los tics se explican por cambios en el funcionamiento frontoestriatal [4], al detectarse variaciones estructurales en los ganglios basales, como la disminución del volumen del núcleo caudado, el putamen y el globo pálido, especialmente en el hemisferio izquierdo [5], así como un hipometabolismo estriatal [6]. La disfunción de los circuitos frontoestriatales (observada en patologías como la enfermedad de Parkinson o el corea de Huntington) afecta a la corteza orbitofrontal, la corteza prefrontal dorsolateral y la corteza cingulada anterior, áreas anatómicas implicadas en funciones cognitivas importantes y que muestran cambios en la actividad y metabolismo en comparación con los niños y adolescentes normales sin TT [7]. La corteza prefrontal dorsolateral está implicada en la flexibilidad cognitiva [8], la memoria [9] y los procesos atencionales [10]. La corteza orbitofrontal se asocia más selectivamente con problemas en tareas de refuerzo y aprendizaje inverso [11]. Por último, la corteza cingulada anterior es importante para la solución de problemas [12] y está activa durante las tareas que implican la inhibición de la respuesta [13]. Los pacientes con TT y TDAH suelen presentar deficiencias en la inhibición de la respuesta [14], en la memoria de trabajo [15] y en la flexibilidad cognitiva [16], mientras que los que muestran comorbilidad clínica con TOC asocian un pobre aprendizaje inverso [17] y un déficit inhibitorio [18].

\section{Funciones motoras, visuomotoras y perceptivas}

Algunos estudios han concluido que los niños con TTSC presentan déficit en la integración visuomotora, en base al rendimiento en el test de integración visuomotora de Beery [19], pero otros no [20].
Unidad de Neuropsicología; Hospital Clínico Universitario de Valencia (R. Espert). Departamento de Psicobiología; Universitat de València; Valencia (R. Espert, M. Gadea, M. Aliño). Hospital General Universitario Santa María del Rosell; Cartagena, Murcia, España (J. Oltra-Cucarella).

Correspondencia:

Dr. Raúl Espert Tortajada. Departamento de Psicobiologia. Universitat de València. Avda. Blasco Ibáñez, 21. E-46010 Valencia.

E-mail:

raul.espert@uv.es

Declaración de intereses: Los autores manifiestan la inexistencia de conflictos de interés en relación con este aertículo.

Aceptado tras revisión externa: 17.01.17.

Cómo citar este artículo:

Espert R, Gadea M, Aliño M, OltraCucarella J. Neuropsicología del trastorno del Tourette: cognición, neuroimagen y creatividad. Rev Neurol 2017; 64 (Supl 1): S65-72.

(c) 2017 Revista de Neurología 
Otros trabajos han evaluado la ejecución en tareas de copia de diseños complejos (p. ej., figura compleja de Rey-Osterrieth), pero con resultados más dispares en niños con comorbilidad con TDAH [21]. Hay más evidencias acerca del déficit en el funcionamiento motor en TT mediante tareas como el Finger Tapping Test [22], el test del tablero de Purdue (Purdue Pegboard Test) [23] o el Grooved Pegboard [22]. Estos trastornos de tipo motor es probable que se deban a la disfunción de los ganglios basales [24], a los cambios en la conectividad interhemisférica o al aumento del tamaño del cuerpo calloso [25]. En resumen, a pesar de haberse publicado trabajos que hablan de un déficit visuomotor y de integración perceptiva, la evidencia de dichos déficits neuropsicológicos en el TTSC es escasa y precisa más investigación.

\section{Atención}

Varios estudios han aportado pruebas acerca de déficits atencionales en el TT, principalmente en tareas de vigilancia como el test del trazo (Trail Making Test) o el test de percepción de sonidos vocales, especialmente en la variante de comorbilidad con TDAH. Sin embargo, es difícil concluir que el TTSC se asocia con dificultades atencionales, dado que pueden ser sutiles o pasar inadvertidas. Estos déficits pueden deberse a la posible influencia de factores como la velocidad motora o la flexibilidad cognitiva [26].

\section{Memoria y aprendizaje}

Algunos estudios destacan la presencia de déficit de memoria en el TT, sobre todo en el apartado de memoria visual $[27,28]$. Sin embargo, el déficit en la memoria verbal es más difícil de probar, especialmente en el subtest de memoria lógica de la escala de memoria de Wechsler revisada. Los estudios neuropsicológicos en TT han proporcionado poca evidencia en alteraciones de aprendizaje de lista de palabras, memoria inmediata y semántica, así como en memoria verbal de reconocimiento [29].

\section{Funciones ejecutivas}

Las pruebas de la función ejecutiva evalúan habilidades como la memoria de trabajo, la fluencia verbal, la planificación, la flexibilidad cognitiva o la inhibición de la respuesta. En este dominio cognitivo tampoco se evidencian de forma clara posibles déficits ejecutivos en el TTSC, excepto en la inhibición de la respuesta [29].

\section{Inhibición}

Los déficits en el test de Hayling en pacientes con TTSC parecen lo suficientemente consistentes como para sugerir que esta patología se asocia con alteraciones en el funcionamiento inhibitorio, independiente de la comorbilidad con TDAH. Sin embargo, pruebas como el test go/no-go o el test de Stroop no han mostrado deterioros claros en esta función [30]. El test de Hayling consiste en completar oraciones en las cuales falta la última palabra. El sujeto debe realizar la tarea en dos condiciones: en la condición A debe producir una palabra que complete de manera coherente la oración, y en la condición $\mathrm{B}$, una palabra no relacionada con la oración. En la condición A, el contexto sintáctico y semántico del estímulo conducen a la activación de una palabra coherente con el campo semántico. En la condición $B$, hay que inhibir la respuesta dominante (la tendencia a completar la oración de manera lógica) y buscar una palabra no relacionada con el contexto sintáctico y semántico que la oración impone. Este test se asocia a una mayor actividad en el giro cingulado anterior [31]. Las vías cingulares anteriores pueden contribuir al desempeño de numerosas tareas, como procesos inhibitorios, atencionales e intencionales, y se han relacionado con procesos de conflicto de respuesta y control de la conducta.

\section{Memoria de trabajo}

Los estudios publicados han mostrado poca evidencia de déficit en la memoria de trabajo verbal en adultos o niños con TTSC en tareas $n$-back, estimación de tiempo o test de span de dígitos [32]. Si se presenta con TDAH comórbido sí se han detectado problemas en esta función ejecutiva usando la prueba de bloques de Corsi [33].

\section{Planificación y multitarea}

Numerosos estudios que han evaluado el desempeño en la planificación mediante pruebas como la torre de Londres o la torre de Hanoi en niños y adultos con TTSC no han detectado déficit alguno [29]. Lo mismo ocurre en los tests de multitarea $[34,35]$. Sin embargo, el TT con TDAH comórbido ha demostrado alteraciones en ambas funciones ejecutivas [36].

\section{Flexibilidad cognitiva}

Esta función parece estar poco afectada en el TTSC, tal y como se demuestra mediante tareas de alternancia de objetos, test de denominación de letras y dígitos o la prueba de clasificación de cartas de Wisconsin [36]. 


\section{Fluencia verbal}

Existe cierta evidencia, aunque débil, de una pobre fluencia verbal en pacientes con TTSC. Puede ser que esta función se asocie con problemas inhibitorios, lo que podría conducir a una reducción de los recursos cognitivos disponibles para la generación de palabras [37].

\section{Estudios de neuroimagen}

La necesidad de una descripción neurofisiológica sistémica que asociara la neurofisiopatología celular con observaciones clínicas del TT ha estado latente durante las últimas décadas. Tras 16 años de investigación mediante paradigmas de activación por medio de tomografía por emisión de positrones y resonancia magnética funcional en estos pacientes, ha sido posible generar un cuerpo empírico basado en la asociación de sintomatología característica del TT con actividad cerebral atípica. A grandes rasgos, dicha activación y diferencias funcionales se han asociado a los ganglios basales y a sus circuitos corticoestriado-talamocorticales en contraste con población no patológica [38-41].

Adentrándonos más en el TT, es necesario realizar una serie de especificaciones conceptuales para el consiguiente entendimiento de este apartado. Resulta esperable el hallazgo de un patrón funcional distinto dependiendo del componente sintomatológico de estudio en este tipo de población: la producción de tics se considera como el componente que se emite externamente, mientras que los impulsos premonitorios se consideran como el componente interno antes de su producción [41]. Partiendo de esta diferenciación conceptual surge la creación de diferentes paradigmas de investigación, los cuales generan a su vez datos y conclusiones complementarios sobre cómo funciona el cerebro de estos pacientes durante cada componente sintomatológico estudiado.

Los hallazgos principales en la última década indican que la producción libre de tics se asocia con activaciones prefrontales, premotoras y subcorticales [42]. Neuner et al [43] alcanzaron a diferenciar entre la activación producida en el momento in situ de la producción de estos tics y el momento previo a su externalización. El área motora suplementaria, región muy investigada en este síndrome, y el opérculo parietal se activaban significativamente dos segundos antes de la producción del tic, coincidiendo con los hallazgos de Fried et al [44], en los que la sensación subjetiva de impulsividad premonitoria se producía cuando los sujetos se some- tían a estimulación eléctrica del área motora suplementaria. Por su parte, un segundo antes del tic se detectó una red de activación que incluía el cíngulo anterior, el putamen, la ínsula, la amígdala, el cerebelo y la corteza occipital. Asimismo, durante la producción de tics in situ, el tálamo, el opérculo central y la corteza motora primaria y somatosensorial eran las estructuras que evidenciaban un incremento de activación. Estos resultados de Neuner et al [43] convergen con los hallados por Worbe et al [45], quienes concluyeron que las áreas primarias motoras y sensoriales, el lóbulo paracentral, el área motora suplementaria y la corteza parietales presentan una mayor conectividad estructural con el estriado y el tálamo. Así pues, tanto en la producción de tics como en los impulsos premonitorios existe una mayor activación y conectividad desde áreas corticales motoras y somatosensoriales a estructuras subcorticales.

Pero la simple detección de estas anomalías funcionales durante la producción de tics debe, a su vez, contrastarse debidamente con respecto a la respuesta cuando se lleva a cabo una supresión voluntaria de los tics. Las regiones cerebrales que evidenciaron mayores niveles de activación en pacientes con TT ante ensayos experimentales basados en un paradigma go/no-go -entendiendo la parte no-go como la supresión de tics- se situaban de forma bilateral en la corteza prefrontal y también en estructuras subcorticales izquierdas (tálamo y caudado) [41]. Heise et al [46] encontraron una hiperexcitabilidad cortical, la cual interpretaron como la manifestación de inputs estriadotalámicos disfuncionales hacia la corteza motora primaria, planteando así la existencia de mecanismos fisiopatológicos compensatorios. En este sentido, durante un estado de reposo, la actividad anormal del bucle estriadotalámico, el cual supone una aferencia hacia áreas corticales motoras, originaría una desinhibición de la corteza motora, facilitando la ocurrencia de los tics. Por el contrario, la hiperactivación durante la ejecución de movimiento por parte de áreas motoras y prefrontales -regiones implicadas en la preparación del movimiento- operaría como un control 'de arriba a abajo' (top-down), suprimiendo los inputs subcorticales aberrantes y, a su vez, la manifestación de tics. Esta explicación referente a los mecanismos compensatorios del propio cerebro es, sin duda, convergente a la obtenida en paradigmas donde se estudia el movimiento voluntario. Sobre todo la corteza prefrontal izquierda también ha demostrado estar sistemáticamente más activa cuando pacientes con TT intentan desarrollar un movimiento volitivo. Este patrón de activación de la corteza prefrontal su- 
giere que este tipo de movimientos suponen un esfuerzo cognitivo mayor para esta clase de pacientes que para el resto de la población [41].

Cuando se analizan los resultados en base a paradigmas de estado de reposo, Worbe et al [45] evidenciaron una conectividad anormalmente elevada por parte del estriado y el tálamo con respecto a las cortezas motora y sensorial primarias, el lóbulo paracentral, el área motora suplementaria y la corteza parietal. Asimismo, Tinaz et al [47] encontraron también una conectividad incrementada entre la corteza sensorimotora y la porción anterior de la ínsula, a la cual se atribuyen sensaciones corporales [48]. Estos resultados sugieren que pacientes con TT presentan un incremento de la conciencia en cuanto a sensaciones corporales, hecho que explicaría el incremento de impulsos premonitorios asociados a aliviar esa sensación del cuerpo y, por tanto, la consiguiente externalización de tics [41].

Por último, algunos estudios han efectuado análisis correlacionales de la gravedad de la sintomatología con patrones de activación cerebral. En un inicio, Baym et al [49] mostraron correlaciones significativas positivas entre la gravedad de los tics y la actividad del tálamo, putamen bilateral, núcleo subtalámico derecho, núcleo dopaminérgico derecho (sustancia negra y área tegmental ventral) y corteza prefrontal bilateral. Por su parte, Neuner et al [43] observaron recientemente que la intensa conectividad del área motora suplementaria y la corteza motora correlacionaba positivamente con la gravedad de los tics y, además, era independiente de la medicación, sexo y edad de los pacientes con TT. Igualmente, la intensidad de los impulsos premonitorios correlacionaba positivamente con la activación en la ínsula derecha y el área motora suplementaria bilateral [50].

\section{Trastorno de Tourette y creatividad}

Las teorías evolucionistas proponen la existencia de aspectos adaptativos en los trastornos mentales que justifican su persistencia en el acervo genético. $\mathrm{Su}$ lógica tiene en cuenta que ciertos trastornos mentales implican una base de vulnerabilidad genética y que dicho genoma es seleccionado expresamente generación tras generación, quizá debido a que otorga ventajas en algunas situaciones, para el individuo o para el grupo. Dichas ventajas pueden rastrearse en los pacientes o familiares, lo que ofrece una oportunidad para comprobar la veracidad de estos postulados [51]. La ventaja adaptativa más estudiada es la creatividad. La conexión entre enfermedad mental y creatividad se puede encontrar des- de muy antiguo, aunque con resultados contradictorios. En 1998, una revisión de 29 estudios sólo encontró evidencia significativa en nueve de ellos; sin embargo, los autores indicaron que, a pesar de esto, se seguía considerando que dicha asociación 'debería existir' [52]. El debate continúa y uno de los acuerdos es que se debe conocer el constructo de estudio (la creatividad) en términos de definición, operacionalización y herramientas de medida apropiadas para poder llegar a conclusiones válidas [53].

La creatividad se define como la capacidad de generar un producto que es a la vez nuevo y apropiado, mientras que el proceso creativo consiste en varias etapas, desde la preparación y la incubación, hasta la inspiración y la verificación [54]. Como factores biológicos predisponentes para ser una persona creativa se contempla una base genética, un factor de masculinidad cerebral y una alta inteligencia (o ser especialmente talentoso para el campo específico en el que se cree), así como ciertas características intrínsecas de personalidad, mientras que como factores externos se contemplan un contexto familiar y social que posibilite el proceso creativo, un alto nivel de entrenamiento en la disciplina ('regla de los 10 años') y la receptividad del campo al que va dirigida la obra [55]. Cuando uno compara estos aspectos con el sufrimiento y el poco control ejecutivo general que muestran los pacientes con un trastorno mental, resulta difícil imaginarse la asociación más allá de por una imaginería romántica [53]. Además, si bien las personas creativas padecen frecuentemente algún tipo de psicopatología, lo contrario no tiene por qué ser cierto: no todas las personas con una enfermedad mental son creativas, y esto probablemente se deba a algún umbral de gravedad de la enfermedad (más allá del cual la manifestación creativa no se produce o no es aceptada como tal por el exterior) o a la falta de alguno de esos ingredientes esenciales comentados anteriormente (p. ej., el talento) [55]. En cuanto al TT, existen ejemplos de artistas notables que se sospecha que pudieron haberlo padecido (aunque se debe ser prudente con los diagnósticos retrospectivos). Sacks comenta el caso del eminente poeta inglés Samuel Johnson, quien 'mostraba rituales y compulsiones, tics, gesticulación exagerada... y un estado de aceleración motora impulsiva e involuntaria, compatible de manera convincente con TT' [56]. Pero es en el ámbito de la creación musical en donde se encuentra el mayor número de artistas, músicos de renombre, aquejados de manera real o con sospecha de padecer TT. El caso más famoso es el de Mozart. La posibilidad de que Mozart cumpliera criterios de TT se propuso en 1983 en el Congreso Mundial de 
Psiquiatría en Viena, basándose en la presencia documentada de tics fonéticos, faciales y corporales, así como en su lenguaje escrito, pues se conservan cartas plenas de palabras soeces y vulgares, relativas a defecación y temas anales en general (coprografías), que podrían encajar en el diagnóstico [57, 58]. Sin embargo, Sacks desconfía del diagnóstico de TT [56] y son varios los autores en la actualidad que critican la falta de evidencia clara al respecto $[59,60]$. Una de las objeciones más interesantes es el hecho de haber encontrado la coprografía pero no coprolalia, lo cual no es típico del TT, forzando el diagnóstico al trasladar las expresiones vocales involuntarias al plano escrito [61]. Otros músicos con diagnóstico retrospectivo de TT son Kurt Cobain, quien fue tratado en su infancia con metilfenidato debido al TDAH y al que se le observaron tics compatibles con TT [62], y Michael Jackson, del cual se ha sugerido que podría presentar características de TOC y posible TT con tics vocales y ruidos en sus canciones y coreografías [55]. Por otra parte, existen varios músicos famosos, vivos, que sí cuentan de facto con un diagnóstico de TT, como Michael Wolf (pianista de jazz y compositor), Nick van Bloss (pianista clásico y compositor) y James Durbin (cantante y guitarrista), lo que sugiere que podría existir una conexión entre la creatividad musical y el TT [63]. En cualquier caso, la pregunta sería si estos autores crean a pesar del TT o si el TT desempeña un papel etiológico en el desarrollo de su creatividad. Según Sacks, dar una respuesta razonable a esta pregunta es complejo porque hay diferentes formas del síndrome. En un extremo está el TT estereotipado, cuya sintomatología es considerada por los pacientes como completamente negativa y en absoluto relacionada con la creatividad. Pero hay otras formas más elaboradas en las que los pacientes destacan por su alegría y extravagancia, y por sus invenciones, dramatizaciones y asociaciones inesperadas, a veces surrealistas, que podrían ayudar a la creatividad musical [56]. De hecho, Sacks cita dos músicos profesionales con TT, virtuosos de la batería, que creían que el TT era la causa de su creatividad musical y utilizaban la música para calmar (o transformar en otra cosa) sus accesos de tics [56]. Quizá aquí resulte conveniente recordar los dos componentes básicos de la creatividad citados anteriormente: la capacidad de producir algo que sea novedoso y a la vez que el producto generado resulte apropiado. Es posible que, en los trastornos mentales, los pacientes sí sean capaces de generar un producto novedoso, pero sólo algunos de ellos sean capaces de ejercer un control ejecutivo eficiente para evaluar lo apropiado de su output ideacional, siendo éstos los reconocidos como creativos [64]. Sería interesante realizar un estudio sobre la prevalencia del TT en la población general frente a artistas o músicos para extraer conclusiones más firmes sobre estas propuestas. Por otra parte, y dada la evidencia anecdótica que publicó Sacks, la hipótesis de utilizar la música como terapia se puso a prueba recientemente. Se comprobó que los tics disminuyen cuando los pacientes están involucrados en actividad musical, mediante cuestionarios que se aplicaron a un grupo de 29 pacientes con TT, encontrándose correlaciones significativas. A continuación se seleccionó a ocho pacientes y se les observó en una variedad de condiciones, y se halló que los tics casi desaparecieron cuando estaban tocando música y que su frecuencia disminuía también cuando estaban simplemente escuchando música o utilizando imágenes mentales de actuaciones musicales [65]. Los autores sugieren que la atención localizada, el control motor fino y la conducta dirigida a una meta (todo ello necesario para la ejecución musical) posibilitaron la desaparición de los tics. Sería de interés que se replicara este experimento por sus implicaciones positivas para el tratamiento de la enfermedad.

\section{Conclusiones}

Como hemos visto, la revisión de los estudios neuropsicológicos en el TTSC apenas detectan deterioro cognitivo, en comparación con patologías neurodegenerativas frontoestriales como la enfermedad de Parkinson o el corea de Huntington, y si lo hay es muy débil y poco consistente. Bornstein [28] sugiere que un subgrupo que comprende aproximadamente sólo el $20 \%$ de los pacientes con TT puro pueden tener un peor rendimiento neuropsicológico. Si los pacientes presentan déficits en dominios cognitivos fundamentales como el aprendizaje o la memoria, es evidente que estas dificultades pueden afectar el rendimiento en una serie de tareas. Los pacientes rendirán peor en tareas complejas porque implican varios componentes ejecutivos, y si los pacientes tienen incluso deficiencias sutiles en componentes múltiples, esto podría contribuir al deterioro del rendimiento cognitivo. Otras dificultades que podrían explicar los resultados inconsistentes pueden derivarse de una evaluación neuropsicológica mediante el uso de datos normativos en lugar de emplear un grupo control. Una variable de confusión en los resultados podría ser la gravedad de los tics, que a menudo fluctúan de forma paralela al rendimiento cognitivo de los pacientes. Otra podría ser la edad de los pacientes cuando son 
evaluados, de modo que los pacientes más jóvenes podrían manifestar una mayor disfunción frontoestriatal, que tiende a normalizarse en la edad adulta en los casos menos graves. Finalmente, existe un mayor riesgo de deterioro cognitivo en niños con diagnóstico de TT con comorbilidades tales como el TDAH o el TOC. En resumen, hay pocas pruebas convincentes de deterioro en el TTSC en habilidades cognitivas ligadas al funcionamiento de la corteza prefrontal dorsolateral, como la fluidez verbal, la planificación, la memoria o la flexibilidad cognitiva, lo que indica que es poco probable una disfunción de las vías frontoestriatales. Podemos concluir que los cambios en los circuitos prefrontales pueden conducir a déficits ejecutivos asociados con síntomas de TT con TDAH, mientras que la disfunción del circuito orbitofrontal puede ocurrir en el TT comórbido con TOC.

A partir de los estudios de neuroimagen funcional realizados a lo largo de la última década ha sido posible sentar una base que pueda explicar la sintomatología clínica de pacientes con TT. Es evidente el papel que desempeña la corteza motora, específicamente, el área motora suplementaria y la corteza cingulada anterior en diferentes aspectos de la ejecución volitiva del movimiento, respuesta de inhibición y producción externa de tics. Asimismo, la hiperactivación cortical por parte de estos pacientes representa la actividad cortical compensatoria y necesaria para mantener los tics y sus impulsos premonitorios bajo control. Por último, la asociación hallada entre la gravedad de la sintomatología y la actividad de la corteza premotora dorsal y del área motora suplementaria ha proporcionado a los datos de resonancia magnética funcional una validez clínica ausente hasta la fecha.

Finalmente, la relación entre creatividad y psicopatología en general dista de estar clara y no parece en absoluto que sea lineal [51]. Una vez asumidas estas limitaciones y teniendo prudencia en la interpretación de los datos, mayormente de tipo observacional y nota histórica, encontramos que la bibliografía propone la existencia de una asociación entre el TT y la creatividad musical. De ser cierto, esto podría dar lugar al desarrollo de programas de intervención clínica basados en la música, cuya utilidad ya ha sido demostrada por algunos autores [65].

\section{Bibliografía}

1. Robertson MM. Tourette syndrome, associated conditions and the complexities of treatment. Brain 2000; 123: 425-62.

2. Khalifa N, Von Knorring AL. Tourette syndrome and other tic disorders in a total population of children: clinical assessment and background. Acta Paediatr 2005; 94: 1608-14.

3. Cavanna AE, Servo S, Monaco F, Robertson MM. The behavioral spectrum of Gilles de la Tourette syndrome. J Neuropsychiatry Clin Neurosci 2009; 21: 13-23.

4. Mink JW. Basal ganglia dysfunction in Tourette's syndrome: a new hypothesis. Pediatr Neurol 2001; 25: 190-8.

5. Singer HS, Reiss AL, Brown JE, Aylward EH, Shin B, Chee E, et al. Volumetric MRI changes in basal ganglia of children with Tourette's syndrome. Neurology 1993; 43: 950-6.

6. Chase TN, Foster NL, Fedio P, Brooks R, Mansi L, Kessler R, et al. Gilles de la Tourette syndrome: studies with the fluorine-18 labeled fluorodeoxyglucose positron emission tomographic method. Ann Neurol 1984; 15: S175.

7. Singer HS. Tourette's syndrome: from behaviour to biology. Lancet Neurol 2005; 4: 149-59.

8. Smith AB, Taylor E, Brammer H, Rubia K. Neural correlates of switching set as measured in fast event related, functional, magnetic resonance imaging. Hum Brain Mapping 2004; 21 : 214-47.

9. Aleman A, Van't Wout M. Repetitive transcranial stimulation over the right dorsolateral prefrontal cortex disrupts digit span task performance. Neuropsychobiology 2008; 57: 44-8.

10. Johnson SA, Strafella AP, Zatorre RJ. The role of the dorsolateral prefrontal cortex in bimodal divided attention: two transcranial magnetic stimulation studies. J Cogn Neurosci 2007; 19: 907-20.

11. Rolls ET. The functions of the orbitofrontal cortex. Brain Cogn 2004; 55: 11-29.

12. Carter CS, Braver TS, Barch DM, Noll D, Cohen JD. Anterior cingulate cortex, error detection and the online monitoring of performance. Science 1998; 280: 747-59.

13. Barch DM, Braver TS, Akbudyak E, Conturo T, Olliger J, Snyder A. Anterior cingulate cortex and response conflict: effects of response modality and stimulus domain. Cereb Cortex 2001; 11: 837-48.

14. Barkley RA. Behavioral inhibition, sustained attention, and executive functions: constructing a unified theory of ADHD. Psychol Bull 1997; 121: 65-94.

15. Murphy KR, Barkley RA, Bush T. Executive functioning and olfactory identification in young adults with attention deficithyperactivity disorder. Neuropsychology 2001; 15: 211-20.

16. Reader MJ, Harris EL, Schuerholz LJ, Denckla MB. Attention deficit hyperactivity disorder and executive function. Dev Neuropsychol 1994; 10: 493-512.

17. Valerius G, Lumpp A, Kuelz AK, Freyer T, Voderholzer U. Reversal learning as a neuropsychological indicator for the neuropathology of obsessive compulsive disorder? A behavioral study. J Neuropsychiatry Clin Neurosci 2008; 20: 210-8.

18. Bannon S, Gonsalvez CJ, Croft RJ, Boyce PM. Response inhibition deficits in obsessive-compulsive disorder. Psychiatry Res 2002; 110: 165-74.

19. Brookshire BL, Butler IJ, Ewing-Cobbs L, Fletcher JM. Neuropsychological characteristics of children with Tourette syndrome: evidence for a nonverbal learning disability? J Clin Exp Neuropsychol 1994; 16: 289-302.

20. Chang SW, McCracken JT, Piacentini JC. Neurocognitive correlates of child obsessive compulsive disorder and Tourette syndrome. J Clin Exp Neuropsychol 2006; 29: 724-33.

21. Harris EL, Schuerholz LJ, Singer HS, Reader MJ, Brown JE, Cox $\mathrm{C}$, et al. Executive function in children with Tourette syndrome and/or comorbid anxiety-deficit hyperactivity disorder. J Int Neuropsychol Soc 1995; 1: 511-6.

22. Bornstein RA, Baker GB, Bazylewich T, Douglass AB. Tourette's syndrome and neuropsychological performance. Acta Psychiatr Scand 1991; 84: 212-6.

23. Bloch MH, Sukhodolsky DG, Leckman JF, Schultz RT. Fine-motor skill deficits in childhood predict adulthood tic severity and global psychosocial functioning in Tourette's syndrome. J Child Psychol Psychiatry 2006; 47: 551-9.

24. Bohnen NI, Kuwabara H, Constantine GM, Mathis CA, Moore RY. Grooved Pegboard Test as a marker of nigrostriatal denervation in Parkinson's disease. Neurosci Lett 2007; 424: 185-9.

25. Plessen KJ, Gruener R, Lundervold A, Hirsch JG, Xu D, Bansal R, et al. Reduced white matter connectivity in the corpus callosum of children with Tourette syndrome. J Child Psychol Psychiatry 2006; 47: 1013-22. 
26. Arbuthnott K, Frank J. Trail Making Test Part B as a measure of executive control: validation using a set-switching paradigm. J Clin Exp Neuropsychol 2000; 22: 518-28.

27. Watkins LH, Sahakian BJ, Robertson MM, Veale DM, Rogers RD, Pickard KM, et al. Executive function in Tourette's syndrome and obsessive-compulsive disorder. Psychol Med 2005; 35: 571-82.

28. Bornstein RA. Neuropsychological performance in adults with Tourette's syndrome. Psychiatry Res 1991; 37: 229-36.

29. Eddy CM, Rizzo R, Cavanna AE. Neuropsychological aspects of Tourette syndrome: a review. J Psychosom Res 2009; 67: 503-13.

30. Eapen V, Crnceca R. Tourette syndrome in children and adolescents: special considerations. J Psychosom Res 2009; 67: 525-32.

31. Nathaniel-James DA, Frith CD. The role of the dorsolateral prefrontal cortex: evidence from the effects of contextual constraint in a sentence completion task. Neuroimage 2002; 16: 1094-9.

32. Channon S, Gunning A, Frankl J, Robertson MM. Tourette's syndrome (TS): cognitive performance in adults with uncomplicated TS. Neuropsychology 2006; 20: 58-65.

33. Poysky J, Khan H, Krull K, Jankovic J. Executive dysfunction and comorbid conditions in Tourette syndrome. Mov Disord 2006; 21: S695-6.

34. Channon S, Crawford S, Vakili K, Robertson MM. Real-lifetype problem-solving in Tourette's syndrome. Cogn Behav Neurol 2003; 16: 3-15.

35. Channon S, Sinclair E, Waller D, Healey L, Robertson MM. Social cognition in Tourette's syndrome: intact theory of mind and impaired inhibitory functioning. J Autism Dev Disord 2004; 34: 669-77.

36. Channon S, Pratt P, Robertson $M$. Executive function, memory and learning in Tourette's syndrome. Neuropsychology 2003; 17: 247-54.

37. Osmon DC, Smerz JM. Neuropsychological evaluation in the diagnosis and treatment of Tourette's syndrome. Behav Modif 2005; 29: 746-83.

38. Leckman JF, Bloch MH, Smith ME, Larabi D, Hampson M. Neurobiological substrates of Tourette's disorder. J Child Adolesc Psychopharmacol 2010; 20: 237-47.

39. Felling RJ, Singer HS. Neurobiology of Tourette syndrome: current status and need for further investigation. J Neurosci 2011; 31: 12387-95.

40. Ganos C, Roessner V, Münchau A. The functional anatomy of Gilles de la Tourette syndrome. Neurosci Biobehav Rev 2013; 37: 1050-62.

41. Zapparoli L, Porta M, Paulesu E. The anarchic brain in action: the contribution of task-based fMRI studies to the understanding of Gilles de la Tourette syndrome. Curr Opin Neurol 2015; 28: 604-11.

42. Stern E, Silbersweig DA, Chee KY, Holmes A, Robertson MM, Trimble M, et al. A functional neuroanatomy of tics in Tourette syndrome. Arch Gen Psychiatry 2000; 57: 741-8.

43. Neuner I, Werner CJ, Arrubla J, Stöcker T, Ehlen C, Wegener HP, et al. Imaging the where and when of tic generation and resting state networks in adult Tourette patients. Front Hum Neurosci 2014; 8: 362-71

44. Fried I, Katz A, McCarthy G, Sass KJ, Williamson P, Spencer SS, et al. Functional organization of human supplementary motor cortex studied by electrical stimulation. J Neurosci 1991; 11 : 3656-66.
45. Worbe Y, Marrakchi-Kacem L, Lecomte S, Valabregue R, Poupon F, Guevara P, et al. Altered structural connectivity of cortico-striato-pallido-thalamic networks in Gilles de la Tourette syndrome. Brain 2015; 138: 472-82.

46. Heise KF, Steven B, Liuzzi G, Thomalla G, Jonas M, MüllerVahl K, et al. Altered modulation of intracortical excitability during movement preparation in Gilles de la Tourette syndrome. Brain 2010; 133: 580-90.

47. Tinaz S, Belluscio BA, Malone P, Van der Veen JW, Hallett MY, Horovitz SG. Role of the sensorimotor cortex in Tourette syndrome using multimodal imaging. Hum Brain Mapp 2014; 35: 5834-46.

48. Simmons WK, Avery JA, Barcalow JC, Bodurka J, Drevets WC, Bellgowan P. Keeping the body in mind: insula functional organization and functional connectivity integrate interoceptive, exteroceptive, and emotional awareness. Hum Brain Mapp 2013; 34: 2944-58.

49. Baym CL, Corbett BA, Wright SB, Bunge SA. Neural correlates of tic severity and cognitive control in children with Tourette syndrome. Brain 2008; 131: 165-79.

50. Tinaz S, Malone P, Hallett M, Horovitz SG. Role of the right dorsal anterior insula in the urge to tic in Tourette syndrome. Mov Disord 2015; 30: 1190-7.

51. Abraham A. Is there an inverted-U relationship between creativity and psychopathology? Front Psychol 2014; 5: 750.

52. Waddell C. Creativity and mental illness: is there a link? Can J Psychiatry 1998; 43: 166-72.

53. Abraham A. Editorial: madness and creativity-yes, no or maybe? Front Psychol 2015; 6: 1055.

54. Sternberg RJ, Lubart TI. The concept of creativity: prospects and paradigms. In Sternberg RJ, ed. Handbook of creativity. Cambridge: Cambridge University Press; 1999. p. 3-15.

55. Blunt SB. The creative brain: fundamental features, associated conditions and unifying neural mechanisms. In Rose FC, ed. The neurology of music. London: Imperial College Press; 2010. p. 31-60.

56. Sacks O. Tourette's syndrome and creativity. BMJ 1992; 305: 1515-6.

57. Simkin B. Mozart's scatological disorder. BMJ 1992; 305: 1563-7.

58. Gunne LM. Hade Mozart Tourettes syndrom? Läkartidningen 1991; 88: 4325-6.

59. Powell H, Kushner HI. Mozart at play: the limitations of attributing the etiology of genius to Tourette syndrome and mental illness. Prog Brain Res 2015; 216: 277-91.

60. Möller HJ. Do features of Mozart's letter-writing style indicate the presence of a neuropsychiatric disorder? Controversies about the Bäsle letters. Eur Arch Psychiatry Clin Neurosci 2014; 264: 367-77.

61. Kammer T. Mozart in the neurological department -who has the tic? Front Neurol Neurosci 2007; 22: 184-92.

62. Cross CR. Mais pesado que o céu: uma biografia de Kurt Cobain. São Paulo: Globo; 2002.

63. Camargo $\mathrm{CH}$, Bronzini A. Tourette's syndrome in famous musicians. Arq Neuropsiquiatr 2015; 73: 1038-40.

64. Fink A, Benedek M, Unterrainer HF, Papousek I, Weiss EM. Creativity and psychopathology: are there similar mental processes involved in creativity and in psychosis-proneness? Front Psychol 2014; 5: 1211.

65. Bodeck S, Lappe C, Evers S. Tic-reducing effects of music in patients with Tourette's syndrome: self-reported and objective analysis. J Neurol Sci 2015; 352: 41-7.

\section{Neuropsychology of Tourette's disorder: cognition, neuroimaging and creativity}

Introduction. Tourette's disorder is the result of fronto-striatal brain dysfunction affecting people of all ages, with a debut in early childhood and continuing into adolescence and adulthood.

Development. This article reviews the main cognitive, functional neuroimaging and creativity-related studies in a disorder characterized by an excess of dopamine in the brain. 
Conclusions. Given the special cerebral configuration of these patients, neuropsychological alterations, especially in executive functions, should be expected. However, the findings are inconclusive and are conditioned by factors such as comorbidity with attention deficit hyperactivity disorder and obsessive-compulsive disorder, age or methodological variables. On the other hand, the neuroimaging studies carried out over the last decade have been able to explain the clinical symptoms of Tourette's disorder patients, with special relevance for the supplementary motor area and the anterior cingulate gyrus. Finally, although there is no linear relationship between excess of dopamine and creativity, the scientific literature emphasizes an association between Tourette's disorder and musical creativity, which could be translated into intervention programs based on music.

Key words. Cognition. Creativity. Dopamine. Functional neuroimaging. Neuropsychology. Tourette disease. 